Paediatr. Paedolog. 2021 - 56 (Suppl 1):S25-S29 https://doi.org/10.1007/s00608-021-00884-8 Angenommen: 8. März 2021

๑ Der/die Autor(en) 2021

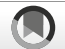

\section{Diabetische Ketoazidose}

Die diabetische Ketoazidose (DKA) ist die Hauptursache für Mortalität und Morbidität von Kindern mit Diabetes mellitus Typ 1 (T1D). Die DKA wird durch einen Insulinmangel und der zusätzlich vermehrten Ausschüttung von kontrainsulinären Hormonen verursacht. Dies führt zu einer verschlechterten peripheren Glukoseverwertung, Glykogenolyse und vermehrter Glukoneogenese, woraus eine Hyperglykämie und Hyperosmolärität resultieren. Die dadurch gesteigerte Lipolyse geht mit der Bildung von Ketonkörpern einher, die in der Folge eine Ketonämie und damit eine metabolische Azidose verursachen. Hyperglykämie und Azidose führen zu einer osmotischen Diurese, die wiederum Dehydratation und Elektrolytverlust zur Folge hat und damit ein prärenales Nierenversagen verursachen kann, das die Azidose zusätzlich verstärkt.

\section{Definition}

Die biochemischen Kriterien der diabetischen Ketoazidose sind:

Hyperglykämie mit Blutglukosewerten über $200 \mathrm{mg} / \mathrm{dl}$, venöser $\mathrm{pH}$-Wert unter 7,3 und/oder Bikarbonatwert unter $15 \mathrm{mmol} / \mathrm{l}$ und/oder Blutketonwert über $3 \mathrm{mmol} / \mathrm{l}$ bzw. mäßige bis starke Ketonurie $[1,2]$.

Je nach Schweregrad kann die DKA weiter unterteilt werden in

- milde DKA (venöser pH-Wert unter 7,3; Bikarbonatwert unter $15 \mathrm{mmol} / \mathrm{L}$ ),

Birgit Rami-Merhar ${ }^{1} \cdot$ Maria Fritsch ${ }^{2} \cdot$ Christine Prchla $^{3} \cdot$ Thomas Hörtenhuber $^{4}$. Elke Fröhlich-Reiterer ${ }^{2}$

${ }^{1}$ Universitätsklinik für Kinder- und Jugendheilkunde, Medizinische Universität Wien, Wien, Österreich

${ }^{2}$ Universitätsklinik für Kinder- und Jugendheilkunde Graz, Klinische Abteilung für Allgemeine Pädiatrie, Medizinische Universität Graz, Graz, Österreich

${ }^{3}$ Abteilung für Kinder- und Jugendheilkunde, Klinik Donaustadt, Wien, Österreich

${ }^{4}$ Abteilung für Kinder- und Jugendheilkunde, Klinik Favoriten, Wien, Österreich

\title{
Akutkomplikationen
}

- mäßige DKA (venöser pH-Wert unter 7,2; Bikarbonatwert unter $10 \mathrm{mmol} / \mathrm{L})$,

- schwere DKA (venöser pH-Wert unter 7,1; Bikarbonatwert unter $5 \mathrm{mmol} / \mathrm{L})$.

\section{Klinische Befunde}

Die Symptome der beginnenden DKA sind zunächst Polydipsie und Polyurie, in weiterer Folge kommt es zusätzlich zu Gewichtsverlust und Leistungs- und Konzentrationsschwäche. Später treten Zeichen der Dehydratation hinzu: Exsikkose, trockene Haut und Schleimhäute, belegte und trockene Zunge. Weitere klinischeZeichen für das Vorliegen einer DKA sind Azetongeruch der Atemluft und eine vertiefte beschleunigte Atmung (Kussmaul- bzw. Azidoseatmung). Auch abdominelle Beschwerden wie typischerweise Übelkeit und Erbrechen treten häufig auf.

\section{Häufigkeit und Risikofaktoren}

Die DKA tritt bei Kindern und Jugendlichen sowohl bei Manifestation der Erkrankung als auch als Komplikation bei bereits bestehendem Diabetes auf.

Die Frequenz der DKA wird in Europa und Nordamerika bei Erstmanifestation zwischen 15-70\% angegeben. Die Inzidenzraten der DKA korrelieren invers mit der regionalen Diabetesinzidenzrate. Das bedeutet, dass Länder mit einer hohen Diabetesinzidenz eine niedrigere DKA-Rate haben. Ebenso wurde gezeigt, dass die Ketoazidoserate bei Erstmanifestation erhöht ist bei kleinen Kindern (unter 4 Jahren), bei Kindern und Jugendlichen ohne Verwandte ersten
Grades mit T1D, Migrationshintergrund und in Familien mit niedrigem sozioökonomischen Status [1, 3-5]. Das Risiko einer DKA bei Patienten mit bekanntem Diabetes beträgt 1-10\%/Patienten/Jahr. Das Risiko ist erhöht bei Kindern mit schlechter Stoffwechselkontrolle oder vorangegangener DKA, jugendlichen Mädchen, Kindern und Jugendlichen mit psychiatrischen Erkrankungen (inklusive Essstörungen) und bei Kindern und Jugendlichen in schwierigen psychosozialen Verhältnissen bzw. mit Migrationshintergrund $[1,6]$. Auch eine Unterbrechung der Insulinzufuhr bei Insulinpumpentherapie (z. B. Pumpenoder Katheterdefekt) kann eine Ursache einer DKA sein $[1,6]$; neuere Daten zeigen jedoch, dass die Frequenz einer ketoazidotischen Entgleisung unter Insulinpumpentherapie rückläufig ist [7].

\section{Morbidität und Mortalität}

Die Mortalitätsraten der DKA ist in nationalen Populationsstudien ziemlich konstant $(0,15-0,31 \%$; [1]). Die Ursache der Mortalität ist fast ausschließlich ein, mit der DKA assoziiertes Hirnödem (57-87\%). Andere Ursachen für Morbidität und Mortalität sind Hypokaliämie, Hyperkaliämie, Hypoglykämie und vaskuläre Zentralnervensystemkomplikationen, wie Thrombosen und Infarkte.

\section{Therapie und Management}

Die Behandlung und Überwachung von Kindern mit DKA sollte grundsätzlich an Abteilungen durchgeführt werden, die über Erfahrung auf diesem Gebiet ver- 
fügen. Die Möglichkeit einer intensiven ärztlichen, pflegerischen und biochemischen Überwachung muss gesichert sein. An diesen Abteilungen sollten klare schriftliche Leitlinien zur DKA-Therapie vorliegen. Die Ziele der Behandlung sind $[1,8]$ :

1. Korrektur der Azidose und der Ketose

2. Korrektur der Dehydratation

3. Normalisierung des Blutzuckerwerts

4. Überwachung bezüglich DKAKomplikationen

5. Identifikation und Behandlung von auftretenden Komplikationen in der Behandlung

Trotz großer Bemühungen, die optimale DKA-Therapie zu finden, bleiben die Daten unschlüssig und es gibt somit keine definitive Therapiestrategie [8].

Prinzipiell müssen sowohl das Flüssigkeits- als auch das Salzdefizit langsam (in 24 bis $48 \mathrm{~h}$ ) ausgeglichen werden. Mit einer intravenösen Flüssigkeitssubstitution sollte unverzüglich, noch vor der Insulintherapie, in Form von 0,9\%iger $\mathrm{NaCl}-L o ̈ s u n g ~ o d e r ~ R i n g e r-L a c t a t ~ b e g o n-$ nen werden. Die benötigte Infusionsmenge pro Tag beträgt meist die 1,5- bis 2-fache Menge des Erhaltungsbedarfs. In den Leitlinien der International Society for Pediatric and Adolescent Diabetes (ISPAD) von 2018 gibt es eine Tabelle, anhand der man den Flüssigkeitsbedarf inklusive $10 \%$ Dehydratation bezogen auf das Körpergewicht ablesen kann. Ein Flüssigkeitsbolus mit $10 \mathrm{ml} / \mathrm{kg} / \mathrm{h}$ wird nicht mehr generell empfohlen, außer bei Vorliegen eines hypovolämischen Schocks $[1,8]$.

\section{Elektrolytsubstitution}

Kalium. Die Serumkaliumkonzentrationen bei Präsentation können normal, erhöht oder erniedrigt sein. Eine Substitution ist immer erforderlich und sollte abhängig von der Serumkaliumkonzentration bei Hypokaliämie sofort, ansonsten spätestens mit Beginn der Insulinsubstitution begonnen werden. Vor Beginn sollte allerdings die Diurese bzw. Nierenfunktion gesichert werden $[1,8]$.

Natrium. Es liegt meist eine Pseudohyponatriämie durch die Hyperglykämie bzw.
Hypertriglyceridämie vor. Eine Substitution ist nur sehr selten notwendig. Bei fehlendem Anstieg sollte an ein Hirnödem gedacht werden.

Chlorid. Im Verlauf ist eine hyperchlorämische Azidose zu erwarten, diese ist allerdings nicht behandlungsbedürftig und sollte nur als Ursache einer sich nicht bessernden Azidose trotz gutem Abfall des Blutzuckerwerts in Erwägung gezogen werden.

Phosphat. Ein Abfall der Phosphatkonzentration ist vor allem nach Beginn der Insulintherapie typisch; eine akute Hypophosphatämie ist selten symptomatisch; Substituierung nur bei einer Phosphatkonzentration unter $0,32 \mathrm{mmol} / \mathrm{l}$ oder Symptomen (Rhadomyolyse, Myopathie - kardial, pulmonal, proximal -, Enzephalopathie).

\section{Azidoseausgleich}

Auch eine schwere DKA ist allein durch die Flüssigkeits- und Insulinsubstitution reversibel. Die Insulinzufuhr stoppt die weitere Ketogenese. Die Behandlung mit Bikarbonat bringt keinen klinischen Benefit, sie kann zu einer paradoxen Zentralnervensystemazidose und $\mathrm{zu}$ einer Hypokaliämie führen und erhöht eventuell sogar die Gefahr des Hirnödems. Deshalb sollte eine Bikarbonatgabe nur in der seltenen Situation einer lebensbedrohlichen Hyperkaliämie erfolgen [1].

Die Rehydratation allein verursacht schon eine Blutzuckersenkung, aber die Insulinsubstitution ist notwendig, um den Blutzuckerwert $\mathrm{zu}$ normalisieren und die Glykogenolyse, Glukoneogenese, Lipolyse und Ketogenese zu unterbrechen und damit die metabolische Azidose auszugleichen.

Eine intravenöse Low-dose-Insulinadministration (empfohlene Dosis ist $0,05$ [bis 1,0$] \mathrm{IE} / \mathrm{kg} / \mathrm{h}$ ) sollte dabei der Standard sein. Der Blutzuckerwert sollte $50-100 \mathrm{mg} / \mathrm{dl} /$ Stunde fallen. Die Insulinsubstitution darf nie ohne gleichzeitige Kaliumsubstitution erfolgen [1,8].

Solange eine metabole Azidose besteht, soll die Insulinzufuhr auch bei einem stärkeren Abfall des Blutzuckerwerts nicht vollständig pausiert wer- den. Bei einem Blutzuckerwert unter $250 \mathrm{mg} / \mathrm{dl}$ ist eine Glukosezufuhr in Form von 5\% Glukoselösung erforderlich. Eine Steigerung auf $10 \%$ oder sogar $20 \%$ Glukoselösung kann bei ausgeprägter Ketoazidose und nur mehr mäßiger Hyperglykämie notwendig sein. Die Tonizität der Lösung soll immer mindestens halbisoton $(0,45 \%)$ sein. Ein kontinuierliches Monitoring und eine genaue Dokumentation müssen durchgeführt werden. Nach dem Ausgleich der Ketoazidose erfolgt der Wechsel auf eine subkutane Insulintherapie $[1,8]$.

\section{Komplikationen}

Mögliche Komplikationen der DKATherapie können folgende sein: inadäquate Rehydratation, Hypoglykämie, Hypokaliämie, hyperchlorämische Azidose und vor allem das Hirnödem.

Das Hirnödem ist eine gefürchtete Komplikation der DKA und tritt typischerweise 4-12 h nach Therapiebeginn auf: Es kann aber auch schon vor Behandlungsbeginn beginnen oder bis $\mathrm{zu}$ $48 \mathrm{~h}$ nach dem Beginn der DKA-Behandlung beobachtet werden. Die Inzidenz des klinisch offensichtlichen Hirnödems liegt bei nationalen Populationsstudien bei $0,5-0,9 \%$ und die Mortalitätsrate bei 21-24\% [1]. Abnormitäten des Mentalstatus (Glasgow Coma Scale unter 14) treten schon bei etwa $15 \%$ der Kinder mit DKA auf [1]. Die Diagnose erfolgt klinisch, eine Bildgebung ist primär nicht notwendig. Als diagnostisch gelten:

- Pathologische motorische oder verbale Reaktion auf Schmerzreiz

- Dekortikations- oder Dezerebrationsstarre

- Hirnnervenausfälle (v. a. III, IV, VI)

- Pathologische Atemmuster (CheyneStokes)

Hinweisend sind folgende zusätzliche Major- und Minorkriterien:

Major:

- Veränderte Bewusstseinslage, Verwirrung, wechselhafte Vigilanz

- Persistierender Herzfrequenzabfall, nicht auf Volumengabe oder Schlafphase zurückzuführen

- Altersinadäquate Inkontinenz 
Minor:

- Erbrechen

- Kopfschmerz

- Somnolenz

- Diastolischer Blutdruck über $90 \mathrm{~mm} \mathrm{Hg}$

- Alter unter 5 Jahre

Zwei Major- oder ein Major- und zwei Minorkriterien gelten als diagnostisch.

Als Risikofaktoren für die Entwicklung eines Hirnödem wiesen Studien jüngeres Alter (unter 5 Jahre), längere Symptomdauer und Diabeteserstmanifestation nach $[1,3,6,8]$.

Bei Verdacht auf ein Hirnödem sollte unmittelbar mit der Behandlung begonnen werden:

Diese besteht aus Hochlagerung des Oberkörpers $\left(30^{\circ}\right)$, Reduktion der Flüssigkeitszufuhr um ein Dirttel sowie Gabe von Mannitol $(0,5-1 \mathrm{~g} / \mathrm{kg})$ über $15 \mathrm{~min}$. Eine Wiederholung ist nach $30 \mathrm{~min}$ möglich.

Nach initialer Therapie kann eine kraniale Bildgebung erfolgen; ein Transfer auf eine Intensivstation sollte bei zunehmender Verschlechterung erwogen werden.

\section{Hypoglykämie}

Das Risiko für Hypoglykämien und die Angst vor Hypoglykämien, sowohl bei den Kindern und Jugendlichen mit T1D als auch bei ihren Eltern, sind noch immer limitierende Faktoren in der Therapie von Kindern mit Diabetes.

Hypoglykämien sind das Resultat eines Missverhältnisses zwischen Insulindosis, Nahrungsaufnahme und Bewegung und die häufigste akute Nebenwirkung der Insulintherapie $[9,10]$.

\section{Definition}

Die Definition der Hypoglykämie ist nicht einheitlich. Die häufigste Definition ist, dass eine Hypoglykämie bei einem Blutzucker unter $70 \mathrm{mg} / \mathrm{dl}$ vorliegt [10]. Man unterscheidet zwischen schweren und milden bzw. moderaten Hypoglykämien [10].

\section{Schwere Hypoglykämie}

Bei Erwachsenen ist diese definiert durch die Notwendigkeit von Fremdhilfe beider Gabe von Kohlenhydraten oder Glukagon. Da Kinder häufig schon aufgrund ihres Alters Hilfe benötigen, ist hier die Definition einer schweren Neuroglykopenie (Koma und/oder Krampfanfall) plus Fremdhilfe.

\section{Milde bzw. moderate Hypoglykämie}

Klinisch gibt es keinen Grund, zwischen einer milden und einer moderaten Hypoglykämie zu unterscheiden, sie werden gleich mit oralen Kohlenhydraten behandelt. Hier kann man noch zwischen symptomatischen (Symptome und Blutzuckerwert $\leq 70 \mathrm{mg} / \mathrm{dl}$ ) und nichtsymptomatischen (Zufallsblutzuckerwert $\leq 70 \mathrm{mg} / \mathrm{dl}$ ohne klinische Symptome) Hypoglykämien unterscheiden.

\section{Häufigkeit und Risikofaktoren}

Milde oder moderate Hypoglykämien können fast täglich auftreten, schwere Hypoglykämien, bei denen der Patient auf Fremdhilfe angewiesen ist, sind selten (5-20 pro 100 Patientenjahre; $[9,10])$. Die Frequenz der schweren Hypoglykämien hat bei Kindern und Jugendlichen in den letzten Jahren aber deutlich abgenommen [11]. Obwohl die Daten teilweise kontrovers diskutiert werden, wurden folgende Risikofaktoren für Hypoglykämien bei Kindern und Jugendlichen mit T1D erarbeitet $[9,10]$ :

- Bei Therapieänderungen zum Alltag (mehr Insulin, weniger Essen, mehr Bewegung)

- Bei jüngeren Kindern

- Bei niedrigen HbA1c-Werten

- Bei wiederholt niedrigen Blutzuckerwerten

- Bei reduzierter Hypowahrnehmung

- Im Schlaf

- Nach Konsum von Alkohol

- Bei längerer Diabetesdauer

\section{Symptome und Hypoglykämie-} wahrnehmung

Kinder und Jugendliche mit Diabetes zeigen eine Vielzahl subjektiver Symptome bei Auftreten einer Hypoglykämie. Es ist üblich, die Hypoglykämiesymptome in zwei Gruppen zu teilen:

1. Autonome Symptome (Hunger, Blässe, Schwitzen, Zittrigkeit, Tachykardie, Unruhe usw.)

2. Neuroglykopenische Symptome (Verwirrtheit, Müdigkeit, Schwindel, Nervosität Kopfschmerzen, Bewusstlosigkeit, Krämpfe usw.)

Jedes Kind hat eigene Hypoglykämiesymptome. Besonders kleinen Kindern (unter 4 Jahren) fehlt oft die Hypoglykämiewahrnehmung oder sie können diese Symptome nicht mitteilen, weshalb diese Patienten ein erhöhtes Risiko für schwere Hypoglykämien haben [9]; dieser Trend wurde in einer rezenten Arbeit (Registervergleich T1D-Patienten USA vs. Deutschland/Österreich unter 6 Jahren) nicht mehr gefunden [12].

\section{Ursachen}

\section{Vermindertes Kohlenhydrat- angebot}

Ein vermindertes Kohlenhydratangebot ist die wahrscheinlich häufigste Ursache für Hypoglykämien. Ursachen dafür können Appetitlosigkeit im Rahmen von Infekten, Ablehnung bestimmter Speisen oder die Weigerung etwas zu essen (Kleinkinder) sein, aber auch die falsche Berechnung des Kohlenhydratgehalts einer Mahlzeit.

Bei Jugendlichen kann es nach Alkoholkonsum zu Hypoglykämien kommen, da Alkohol erstens die die Hypoglykämiewahrnehmung beeinträchtigt und zweitens die Glukoneogenese in der Leber hemmt $[10,13]$.

Intensive körperliche Anstrengung Ausgeprägte oder langanhaltende körperliche Belastung können Hypoglykämien zur Folge habe, wenn sie nicht vorsorglich durch Sport-Broteinheiten und/ oder verminderte Insulinabgabe kompensiert werden. Typisch und gefürchtet bei Kindern und Jugendlichen ist auch eine verzögerte, mehre Stunden nach körperlicher Anstrengung auftretende Hypoglykämie [10]. 


\section{Verstärkte Insulinwirkung}

Ursachen für eine verstärkte Insulinwirkung sind Verwechseln der Insulinpräparate (z. B. lang- mit kurzwirksamen Insulin), falsches Ablesen der Insulinmenge in der Spritze oder beim Pen und ungenügendes Schwenken von trüben Insulinen oder eine fehlerhafte Injektionstechnik (z. B. Injektion in die Muskulatur oder ein Blutgefäß; [10]). Weitere Risikofaktoren sind: eine gestörte Hypoglykämiewahrnehmung, eine vorangegangene schwere Hypoglykämie und eine längere Diabetesdauer [10].

\section{Therapie}

Das Ziel ist, den Blutzucker wieder in den Normalbereich anzuheben (über $70 \mathrm{mg} / \mathrm{dL} ;[8,9])$.

\section{Bei leichten Hypoglykämien}

- Orale Gabe von 5 bis 15 g Glukose (Traubenzucker oder Saft), laut ISPAD 0,3 g/kg Körpergewicht (etwa $9 \mathrm{~g}$ bei $30 \mathrm{~kg}$ Körpergewicht, etwa $15 \mathrm{~g}$ bei $50 \mathrm{~kg}$ Körpergewicht; [10])

- Bei Nichtansteigen des Blutzuckerwerts nach 10-15 min Wiederholung der oben angeführten Therapie

- Die Menge der verabreichten Kohlenhydrate hängt von Alter und Gewicht des Kindes und dem glykämischen Index der verabreichten Kohlenhydrate ab [10].

\section{Schwere Hypoglykämien}

- Glukagon i.m. oder s.c. (0,5-1 mg)

- Seit Herbst 2020 steht auch in Österreich ein intranasales Glukagon zur Verfügung

- Nach Restitution Verabreichung von Kohlenhydraten per os (!).

- Glukose i.v. (im Krankenhaus oder Rettungssetting): 10-20\% Glukose, insgesamt 200-500 mg/kg Glukose

In der Erholungsphase nach schwerer Hypoglykämie müssen regelmäßige, enge Blutzuckerkontrollen durchgeführt werden [10].

\section{Prävention}

Die Diabetesschulung des gesamten Umfelds ist essenziell in der Präven- tion der Hypoglykämie (Schulung der Symptome, Risikofaktoren, Blutzuckermessung). Das Ziel der Diabetestherapie ist es, einen Blutzuckerspiegel unter $70 \mathrm{mg} / \mathrm{dl}$ und dabei eine möglichst normoglykämische Einstellung ohne schwere Hypoglykämien zu erreichen. Ein Traubenzucker sollte immer verfügbar sein, ebenso das Blutzuckermessgerät, um eine Hypoglykämie zu bestätigen und sicher behandeln zu können.

Seit dem häufigen Einsatz der kontinuierlichen Glukosemessung wurde die Frequenz der schweren Hypoglykämie weiter reduziert $[9,14,15]$; weitere Erfolge wurden durch eine sensorunterstützte Insulinpumpentherapie mit Hypoabschaltung erzielt $[10,14]$. Die nächste Verbesserung wurde durch HybridClosed-Loop-Systeme erzielt [14].

Neuere Studien mit sensorunterstützter Pumpentherapie zeigten eine Reduktion der Hypoglykämien; insbesondere bei Auftreten von schweren Hypoglykämien und/oder schlechter Hypoglykämiewahrnehmung sollte diese Therapie empfohlen werden [14].

\section{Korrespondenzadresse}

\section{Birgit Rami-Merhar}

Universitätsklinik für Kinder- und Jugendheilkunde, Medizinische Universität Wien

Wien, Österreich

birgit.rami@meduniwien.ac.at

Funding. Open access funding provided by Medical University of Vienna.

\section{Einhaltung ethischer Richtlinien}

Interessenkonflikt. B. Rami-Merhar, M. Fritsch, C. Prchla, T. Hörtenhuber und E. Fröhlich-Reiterer geben an, dass kein Interessenkonflikt besteht.

Für diesen Beitrag wurden von den Autoren keine Studien an Menschen oder Tieren durchgeführt. Für die aufgeführten Studien gelten die jeweils dort angegebenen ethischen Richtlinien.

Open Access. Dieser Artikel wird unter der Creative Commons Namensnennung 4.0 International Lizenz veröffentlicht, welche die Nutzung, Vervielfältigung, Bearbeitung, Verbreitung und Wiedergabe in jeglichem Medium und Format erlaubt, sofern Sie den/die ursprünglichen Autor(en) und die Quelle ordnungsgemäß nennen, einen Link zur Creative Commons Lizenz beifügen und angeben, ob Änderungen vorgenommen wurden.
Die in diesem Artikel enthaltenen Bilder und sonstiges Drittmaterial unterliegen ebenfalls der genannten Creative Commons Lizenz, sofern sich aus der Abbildungslegende nichts anderes ergibt. Sofern das betreffende Material nicht unter der genannten Creative Commons Lizenz steht und die betreffende Handlung nicht nach gesetzlichen Vorschriften erlaubt ist, ist für die oben aufgeführten Weiterverwendungen des Materials die Einwilligung des jeweiligen Rechteinhabers einzuholen.

Weitere Details zur Lizenz entnehmen Sie bitte der Lizenzinformation auf http://creativecommons.org/ licenses/by/4.0/deed.de.

\section{Literatur}

1. Wolfsdorf JI, Glaser N, Agus M, Fritsch M, Hanas R, Rewers A et al (2018) ISPAD Clinical Practice Consensus Guidelines 2018: Diabetic ketoacidosis and the hyperglycemichyperosmolarstate. Pediatr Diabetes 19:155-177

2. Dunger DB, Sperling MA, Acerini CL, Bohn DJ, Daneman D, Danne TPA et al (2004) ESPE/LWPES consensus statement on diabetic ketoacidosis in children and adolescents. Arch Dis Child 89:188-194

3. Fritsch M, Schober E, Rami-Merhar B, Hofer S, Fröhlich-Reiterer $E$, Waldhoer T, Austrian Diabetes Incidence Study Group (2013) Diabetic ketoacidosis at diagnosis in Austrian children: a population-based analysis, 1989-2011. J Pediatr 163(5):1484-8.e1. https://doi.org/10.1016/j. jpeds.2013.06.033

4. Schober E, Rami B, Waldhoer T (2010) Diabetic ketoacidosis at diagnosis in Austrian children in 1989-2008: a population-based analysis. Diabetologia 53:1057-1061

5. Cherubini V, Grimsmann JM, Åkesson K, Birkebæk NH, Cinek O, Dovč K, Gesuita R, Gregory JW Hanas R, Hofer SE, Holl RW, Jefferies C, Joner G, King BR, Mayer-Davis EJ, Peña AS, Rami-Merhar B, Schierloh U, Skrivarhaug T, Sumnik Z, Svensson J, Warner JT, Bratina N, Dabelea D (2020) Temporal trends in diabetic ketoacidosis at diagnosis of paediatric type 1 diabetes between 2006 and 2016: results from 13 countries in three continents. Diabetologia 63(8):1530-1541. https://doi.org/ 10.1007/s00125-020-05152-1

6. Fritsch M, Rosenbauer J, Schober E, Neu A, Placzek K, Holl RW (2011) Predictors of diabetic ketoacidosis in children and adolescents with type 1 diabetes. Experience from a large multicentre database. Pediatr Diabetes 12:307-312

7. Karges B, Schwandt A, Heidtmann B, Kordonouri O, Binder E, Schierloh U et al (2017) Association of insulin pump therapy vs insulin injection therapy with severe hypoglycemia, ketoacidosis, and glycemic control among children, adolescents, and young adults with type 1 diabetes. JAMA J Am Med Assoc 318:1358-1366

8. Kuppermann N, Ghetti S, Schunk JE, Stoner MJ, Rewers A, McManemy JK et al (2018) Clinical trial of fluid infusion rates for pediatric diabetic ketoacidosis. NEngl J Med 378:2275-2287

9. Ly TT, Maahs DM, Rewers A, Dunger D, Oduwole A, Jones TW (2014) Assessment and management of hypoglycemia in children and adolescents with diabetes. Pediatr Diabetes 15:180-192

10. Abraham MB, Jones TW, Naranjo D, Karges B, Oduwole A, Tauschmann M et al (2018) ISPAD Clinical Practice Consensus Guidelines 2018: assessment and management of hypoglycemia in 
children and adolescents with diabetes. Pediatr Diabetes 19:178-192

11. Rosenbauer J, Dost A, Karges B, Hungele A, Stahl A Bächle C et al (2012) Improved metabolic control in children and adolescents with type 1 diabetes: A trend analysis using prospective multicenter data from Germany and Austria. Diabetes Care 35:80-86

12. Maahs DM, Hermann JM, DuBose SN, Miller KM, Heidtmann B, DiMeglio LA et al (2014) Contrasting the clinical care and outcomes of 2,622 children with type 1 diabetes less than 6 years of age in the United States T1D Exchange and German/Austrian DPV registries. Diabetologia 57:1578-1585

13. Cryer PE (2006) Mechanisms of sympathoadrenal failure and hypoglycemia in diabetes. J Clin Invest 116:1470-1473

14. Sherr JL, Tauschmann M, Battelino T, de Bock M, Forlenza G, Roman R et al (2018) ISPAD clinical practice consensus guidelines 2018: diabetes technologies. Pediatr Diabetes 19:302-325

15. Biester T, Grimsmann JM, Heidtmann B, RamiMerhar B, Ermer U, Wolf J, Freff M, Karges B, Agena D, Bramlage P, Danne T, Holl RW (2021) Intermittently Scanned Glucose Values for Continuous Monitoring: Cross-Sectional Analysis of Glycemic Control and Hypoglycemia in 1809 Children and Adolescents with Type 1 Diabetes. Diabetes Technol Ther 23(3):160-167. https://doi.org/10.1089/ dia. 2020.0373

Hinweis des Verlags. Der Verlag bleibt in Hinblick auf geografische Zuordnungen und Gebietsbezeichnungen in veröffentlichten Karten und Institutsadressen neutral.

Hier steht eine Anzeige.

\section{Springer}

